\title{
Visual acuity of dentists under simulated clinical conditions
}

\author{
Martina Eichenberger • Philippe Perrin • \\ Klaus W. Neuhaus · Ueli Bringolf • Adrian Lussi
}

Received: 4 January 2012 / Accepted: 14 May 2012 / Published online: 26 May 2012

(C) Springer-Verlag 2012

\begin{abstract}
Objectives This study examined the near visual acuity of dentists in relation to age and magnification under simulated clinical conditions.

Materials and methods Miniaturized visual tests were performed in posterior teeth of a dental phantom head in a simulated clinical setting (dental chair, operating lamp, dental mirror). The visual acuity of 40 dentists was measured under the following conditions: (1) natural visual acuity, distance of $300 \mathrm{~mm}$; (2) natural visual acuity, free choice of distance; (3) Galilean loupes, magnification of $\times 2.5$; (4) Keplerian loupes, $\times 4.3$; (5) operating microscope, $\times 4$, integrated light; (6) operating microscope, $\times 6.4$, integrated light.

Results The visual acuity varied widely between individuals and was significantly lower in the group $\geq 40$ years of age ( $p<$ $0.001)$. Significant differences were found between all tested conditions $(p<0.01)$. Furthermore, a correlation between visual acuity and age was found for all conditions. The performance with the microscope was better than with loupes even with comparable magnification factors. Some dentists had a better visual acuity without optical aids than others with Galilean loupes.

Conclusions Near visual acuity under simulated clinical conditions varies widely between individuals and decreases
\end{abstract}

M. Eichenberger $\cdot$ P. Perrin $(\bowtie) \cdot$ K. W. Neuhaus $\cdot$ A. Lussi

Department of Preventive, Restorative and Pediatric Dentistry,

School of Dental Medicine, University of Bern,

Bern, Switzerland

e-mail: philippe.perrin@zmk.unibe.ch

U. Bringolf

Nordstrasse 26,

8200 Schaffhausen, Switzerland throughout life. Visual deficiencies can be compensated for with optical aids.

Clinical relevance Newly developed miniaturized vision tests have allowed, in a clinically relevant way, to evaluate the influence of magnification and age on the near visual acuity of dentists.

Keywords Magnification systems · Dental loupes · Operating microscope $\cdot$ Restorative dentistry $\cdot$ Presbyopia

\section{Introduction}

The use of optical aids to improve manual precision has long been a tradition in many medical professions [1]. Dentistry seems to be an ideal environment for the use of loupes and microscopes, because operative treatments are performed in a small and narrow environment. However, the idea that magnification devices should be used as standard devices in dentistry is relatively new [2-4].

The body of evidence supporting the general assumption that optical magnification improves the quality of caries detection and filigree therapy is weak [5]. Most publications about visual acuity and the influence of magnification [6-9], or concerning microscopes and endodontics [2, 10-12], are based on expert opinions and case reports. An obvious gap between anecdotal conjecture and solid evidence-based decision making exists with respect to the use of optical aids in dentistry.

In a previous study [5] with the help of miniaturized visual tests and a light box, the present authors found a large variability in the near visual acuity of dentists and a significant correlation with the dentists' age. Yet, the tests were not incorporated in an oral cavity, and thus, the clinical relevance of these miniaturized visual tests was limited. 
Therefore, the aims of the present study were:

1. To test near visual acuity and the influence of magnification and age in a simulated clinical situation

2. To compare the results to the previous study, where the same group of dentists was tested under standardized conditions ex ore with a light box

\section{Materials and methods}

The near visual acuity of 40 dentists from the Dental School of the University of Bern was evaluated using miniaturized visual tests fixed in a dental phantom head unit. The distribution of the dentists in the different age groups was as follows: $25-30$ years, $n=16 ; 31-35$ years, $n=9 ; 36-40$ years, $n=2 ; 41-$ 45 years, $n=5$; $46-50$ years, $n=1 ; 51-55$ years, $n=3 ; 56-$ 60 years, $n=3 ; 61-65$ years, $n=1$.

Visual tests with E-optotypes, ranging from 0.05 to $0.5 \mathrm{~mm}$, allowed for the quantification of visual acuity at a typical dental working distance, as described in a previous study [5]. The tests were mounted in cavities in maxillary second premolars and first molars of a dental phantom unit (KaVo, Biberach, Germany) (Fig. 1).

The head was positioned on a dental chair. Dental mirrors (Rhodium Nr. 4, Hahnenkratt GmbH, Königsbach, Germany) were used, and the operating lamp (Delight, Planmeca OY, Helsinki, Finland) for conditions 1 through 4 was individually arranged by the test persons.

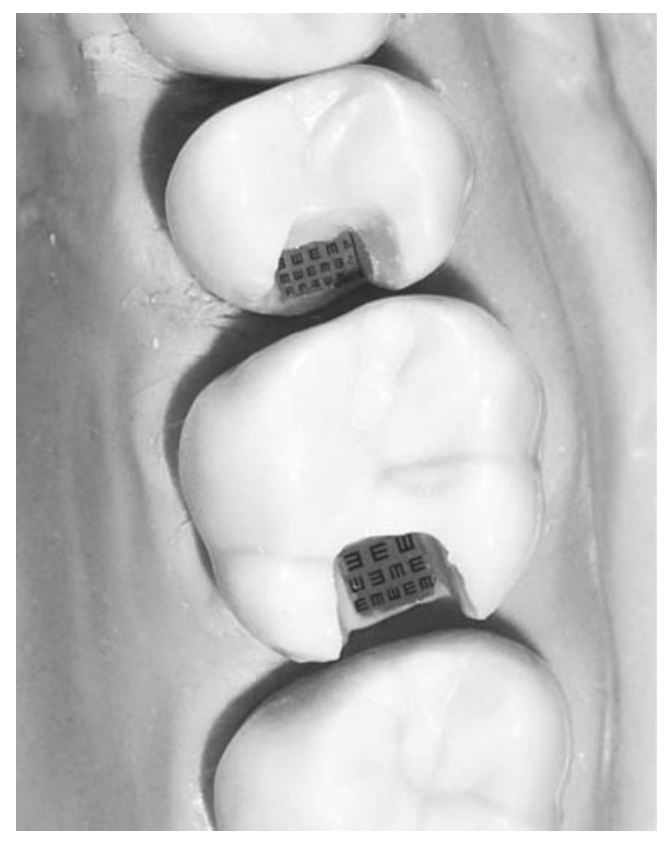

Fig.1 The fixation of the visual tests in distal box cavities of phantom teeth allows the testing of visual acuity under simulated clinical conditions
Visual performance was tested under the following conditions:

1. Natural visual acuity (NV): no magnification devices, distance of $300 \mathrm{~mm}$ (typical working distance), operating lamp

2. Free natural visual acuity (NVf): no magnification devices and free choice of the distance (typical controlling distance), operating lamp

3. Galilean loupe system (G) (EVC250N, SurgiTel, Ann Arbor, MI, USA): magnification of $\times 2.5$; distance of $380 \mathrm{~mm}$; operating lamp

4. Keplerian loupe system (K) (EyeMag Pro S, Zeiss, Oberkochen, Germany): magnification of $\times 4.3$; distance of $400 \mathrm{~mm}$; operating lamp

5. Operating microscope (M4×) (Leica M300, Leica Microsystems, Heerbrugg, Switzerland): magnification of $\times 4$; distance of $250 \mathrm{~mm}$; integrated light

6. Operating microscope (M6.4×): same as condition 5, but with magnification of $\times 6.4$.

The loupes were fixed on a headband, which allowed the dentists to wear their respective prescription eyeglasses (when necessary) in all groups. During the visual tests, the position of the loupes, the eye-object distance, and the reading of the E-optotypes were all controlled by the same expert. The smallest line of the visual test that could be read without mistakes was registered.

The distance between the three bars of the letter E $(=1 / 5$ the size of E) corresponds to the smallest detectable structure [13] and allows to calculate the visual acuity. The metric dimension of the bar spacing in the registered line (e.g., $0.03 \mathrm{~mm}$ ) was converted into the reciprocal value (e.g., 33.3/mm). This allowed to achieve a positive correlation between the metric value and the quality of the visual performance. The influence of the different magnification systems (conditions 1-6) was tested for all dentists. To investigate the influence of the dentists' age, the present authors calculated the correlation between age and visual acuity for the different optical conditions and compared the results of the two subgroups $\mathrm{A}<40$ years $(n=27)$ and $\mathrm{B} \geq 40$ years $(n=13)$. Finally, the results of the present study were compared with the results of the previous study, where the visual performance of the same dentists was tested on the light box and not in a setup simulating the clinical situation.

For statistical analysis, the software $\mathrm{R}$ version 2.13.0 (www.r-project.org) was used. The significance level was set at $\alpha=0.05$. Descriptive statistical analysis was carried out to determine the means and medians as well as the nonparametric $95 \%$ confidence intervals (CI) for all dentists. The influence of the respective groups of dentists and different loupe systems on the visual acuity was analyzed by using rank tests for linear models based on Wilcoxon scores [14], followed by Wilcoxon signed rank tests with 
Bonferroni-Holm correction for multiple comparisons as post hoc tests. Spearman's rank correlation coefficient was used to detect monotone relationships between age and visual acuity. For the third part, Spearman's rank correlation coefficient and Pearson's correlation coefficient were used to detect monotone and linear relationships between the results of the previous study and those of the present one concerning NV, G, and $\mathrm{K}$.

\section{Results}

The mean visual performance of the tested devices is presented in Table 1. The relative improvement of visual performance with magnification ranged from $250 \%$ (Galilean loupe) to $961 \%$ (operating microscope, $\times 6.4$ ), compared to natural visual acuity. Statistical analysis revealed significant differences between all tested conditions $(p<0.01)$ (Table 1$)$.

The correlation between age and visual performance under the different conditions is presented in Table 2. Spearman's rank correlation shows medium correlation values for most of the conditions. The exceptions were NVf with a strong correlation and M6.4× with a weak correlation.

The mean visual performance, medians, and $95 \%$ CIs for groups A ( $<40$ years) and B ( $\geq 40$ years) are presented in Table 3. For all tested conditions, significant differences between the two groups were detected $(p<0.001$ or $p<$ 0.05 ). Both groups benefitted from the magnification of the Galilean and Keplerian loupes and even more from the sophisticated optics of the operating microscope. The visual acuity of group A in natural vision at a closer distance (NVf) was better than the acuity of group B with Galilean loupes (G). The difference between conditions NVf and G was not as pronounced for group A as for group B.

Table 1 Visual performance (mean, median, nonparametric $95 \%$ confidence intervals) and the relative improvement of all tested dentists under all test conditions

\begin{tabular}{lrrlc}
\hline $\begin{array}{l}\text { Condition } \\
(1 / \mathrm{mm})\end{array}$ & $\begin{array}{l}\text { Mean } \\
(1 / \mathrm{mm})\end{array}$ & $\begin{array}{l}\text { Median } \\
(1 / \mathrm{mm})\end{array}$ & $\begin{array}{l}95 \% \mathrm{CI} \\
(1 / \mathrm{mm})\end{array}$ & $\begin{array}{l}\text { Improvement of } \\
\text { visual performance } \\
(\mathrm{NV}=100 \%)\end{array}$ \\
\hline $\mathrm{NV}^{*}$ & 8.43 & 11.52 & $11.52-13.98$ & $100 \%$ \\
$\mathrm{NVf}^{*}$ & 15.20 & 18.04 & $16.79-21.01$ & $180 \%$ \\
$\mathrm{G}^{*}$ & 21.07 & 21.01 & $19.52-22.90$ & $250 \%$ \\
$\mathrm{~K}^{*}$ & 31.94 & 34.34 & $29.76-34.34$ & $379 \%$ \\
M4** & 57.27 & 53.48 & $53.48-58.19$ & $679 \%$ \\
M6.4×* & 81.01 & 81.59 & $75.13-87.41$ & $961 \%$ \\
\hline
\end{tabular}

Note the difference between NV and NVf due to the natural magnification by moving closer to the object

${ }^{*} p<0.01$, significant differences within the columns
Table 2 The correlation between age and visual performance was medium for all optical conditions except NVf (strong correlation) and M6.4× (weak correlation)

\begin{tabular}{lr}
\hline Condition & $\begin{array}{l}\text { Spearman's rank } \\
\text { correlation }\end{array}$ \\
\hline $\mathrm{NV}$ & -0.543 \\
$\mathrm{NVf}$ & -0.747 \\
$\mathrm{G}$ & -0.686 \\
$\mathrm{~K}$ & -0.563 \\
M4× & -0.485 \\
M6.4× & -0.286 \\
\hline
\end{tabular}

The comparison of the present study with the previous study on a light box [5] revealed a strong correlation between the two setups when no magnification device was used (NV; Spearman's correlation coefficient, 0.698) and when the Galilean system was used (G; Pearson's correlation coefficient, 0.725$)$. The correlation was medium for the Keplerian system (K; Spearman's correlation coefficient, 0.622).

\section{Discussion}

A large variability in natural visual acuity was found (0$18.04,1 / \mathrm{mm}$ bar spacing), which corroborated the results of a previous study under optimal conditions ex ore [5]. Some of the tested dentists had better visual acuity without optical aids than others with Galilean loupes. The visual acuity can, as expected, be significantly improved by the use of magnification devices (250-961\%, Table 1), independent of age and natural visual acuity. When using Keplerian loupes, all dentists achieved a significantly higher visual acuity than with Galilean loupes. This could be expected due to the magnification factor. The different magnification factors of the two systems have a clinical rationale: Galilean loupes are small and ergonomic but have a magnification limit of $\times 2.5$ ( $\times 3.2$ with optical constraints), while the magnification of Keplerian loupes is not constrained. Keplerian loupes therefore provide an advantage at a magnification of $\times 3.5$ and above only.

The large difference between the microscope and the head-mounted loupe systems, even with comparable magnification factors, was unexpected. The highly superior performance of the operating microscope could be due to the different optical construction of the microscope with a greater angle between the two optical beams. A further, possibly even more important reason could be the static position of the microscope, which offsets any disturbances caused by head movements. This effect is only detectable in a simulated clinical setting as used in this study.

Presbyopia (loss of accommodation) starts around the age of 40 years and is combined with other changes in the eye, such as decreasing sensitivity to contrast, increasing sensitivity 
Table 3 Visual performance (mean, median, nonparametric $95 \%$ confidence intervals) for the two groups $<40$ and $\geq 40$ years under all test conditions

\begin{tabular}{|c|c|c|c|c|c|c|}
\hline \multirow[t]{2}{*}{ Condition } & \multicolumn{3}{|c|}{ Age $<40$ years $(n=27)(1 / \mathrm{mm})$} & \multicolumn{3}{|c|}{ Age $\geq 40$ years $(n=13)(1 / \mathrm{mm})$} \\
\hline & Mean & Median & $95 \% \mathrm{CI}$ & Mean & Median & $95 \% \mathrm{CI}$ \\
\hline $\mathrm{NV}^{*}$ & 11.16 & 11.52 & $11.52-14.63$ & 3.35 & 0 & n.a. \\
\hline NVf* & 20.29 & 21.01 & $18.27-21.01$ & 5.75 & 0 & n.a. \\
\hline $\mathrm{G}^{*}$ & 23.38 & 21.01 & $21.01-25.38$ & 16.78 & 18.04 & $14.78-18.04$ \\
\hline $\mathrm{K}^{*}$ & 34.38 & 34.34 & $32.05-37.01$ & 27.39 & 27.28 & $24.80-29.76$ \\
\hline $\mathrm{M} 4 \times * *$ & 60.07 & 58.19 & $55.49-64.61$ & 52.06 & 48.08 & $48.08-55.49$ \\
\hline M6.4×** & 85.15 & 87.41 & $82.59-94.21$ & 73.31 & 75.76 & $62.84-81.59$ \\
\hline
\end{tabular}

Note the differences from NVf to G between the two groups

n.a. Not available: due to the skewed distribution, a calculation of the $95 \%$ confidence interval was not possible (in the older group when visual performance with the naked eye was tested

${ }^{*} p<0.001 ; * *<0.05$, significant differences within the columns

to glare, and lower visual performance $[15,16]$. The influence of presbyopia on the visual performance was analyzed in two different mathematical approaches. Spearman's rank correlation confirmed a decrease in the visual acuity over time. The strong correlation between age and natural visual acuity in a free, i.e., closer, distance (NVf) could be expected due to the loss of accommodation. However, the only marginal correlation between age and acuity using the microscope (M6.4×) was unexpected. An explanation could be the fact that the microscope allows a parallel view without any accommodation and is therefore less able to account for presbyopic deficiencies.

The division into two groups $(<40$ and $\geq 40$ years old) allowed the quantification of the age-related deficiencies in a second, more comprehensible approach. The decrease in visual performance is important. In the group $\geq 40$ years old, the condition NVf exhibited a $72 \%$ lower visual performance. This condition has an evident clinical importance: by reducing the working distance, the dentist profits from a natural magnification which is quantified in the difference between the conditions NV and NVf in the younger group A ( $<40$ years old) (Table 3). This natural magnification is widely used in diagnostic situations and mostly compromised with increasing age.

The comparison of the extraoral visual test under optimum visual conditions (direct view, light box, supported head) with the intraoral visual test in a phantom head showed a strong correlation for unaided natural vision and Galilean loupes. The correlation for Keplerian loupes was less pronounced. This could indicate that head-mounted higher magnifications are more sensitive to tremors arising from the head and dental mirrors in a clinical situation. However, an extraoral near vision test seems to be a valid surrogate to judge the performance that would be achieved in the clinical situation.

Common near vision tests, such as those used by opticians, are physically too large to assess visual acuity at the scale on which dentists work [5]. This is, with one exception [17], also true for the few research studies identified in the literature $[15,18,19]$. The E-optotypes used in the present study ranged from 0.05 to $0.5 \mathrm{~mm}$ and were proven to have adequate discriminatory properties [5]. The placement of the tests in artificial teeth provided a realistic simulation of the dental setting, including variables such as the illumination source and the reduced contrast in a cavity, which may influence the visual performance during dental work.

The most objective value for visual performance is the visual acuity angle [13]. However, not all parameters for an objective optical value can be standardized in a simulated clinical setting. The pragmatic question of whether a small structure can be recognized or not seems to be more adequate for a clinical approach. Therefore, the visual acuity angle was substituted for the metric dimension of the smallest recognized bar spacing under the given conditions. The reciprocal calculation of bar spacing allowed for a positive correlation between metric values and visual performance.

The results of this study suggest the importance of reliable near vision tests for dental personnel. Dental students as well as dental personnel with increasing age should be aware of their natural near vision in order to choose adequate optical aids. This also holds true for other medical branches. Whether or not an adjusted visual acuity has an impact on the precision of diagnostic and operative procedures remains the subject of further studies.

\section{Conclusion}

The near visual acuity under simulated clinical conditions varies highly between individuals and decreases over one's lifetime. This decrease is most pronounced in natural vision without magnification devices. There is a need for a free accessible visual test at a dental working distance to objectively measure the visual performance of dental students and dental personnel in order to provide personalized recommendations 
for the use of magnification devices. Individuals with poor natural visual acuity or an age $\geq 40$ years can reliably compensate their visual deficiencies by using optical aids.

Acknowledgments The authors would like to acknowledge Philippe Ducreux (Lunea-Group, France), Prof. Dr.-Ing. Andreas Ettemeyer (NTB Interstaatliche Hochschule für Technik Buchs, Buchs, Switzerland), Martin Roost (Roost-Optik, Schaffhausen, Switzerland), and René Seiler (Fischer \& Loeliger Optik, Bern, Switzerland) for their support with optical issues. The authors also thank Stefanie Hayoz (Institute of Mathematical Statistics and Actuarial Science, University of Bern, Switzerland) for the statistical analysis.

Conflict of interest The authors declare that they have no conflict of interest.

\section{References}

1. Zeiss C (2006) Das Magazin von Carl Zeiss. Innovation 17 (September 2006)

2. Friedman M, Mora AF, Schmidt R (1999) Microscope-assisted precision dentistry. Compend Contin Educ Dent 20:723-728, 730-721, 735-726; quiz 737

3. Friedman MJ (2004) Magnification in a restorative dental practice: from loupes to microscopes. Compend Contin Educ Dent 25:48, $50,53-45$

4. Carr GB (1992) Microscopes in endodontics. J Calif Dent Assoc 20:55-61

5. Eichenberger M, Perrin P, Neuhaus KW, Bringolf U, Lussi A (2011) Influence of loupes and age on the near visual acuity of practicing dentists. J Biomed Opt 16:035003
6. Forgie AH (2001) Magnification: what is available, and will it aid your clinical practice? Dent Update 28(125-128):130

7. Millar BJ (1998) Focus on loupes. Br Dent J 185:504-508

8. Perrin P, Jacky D, Hotz P (2000) The operating microscope in dental general practice. Schweiz Monatsschr Zahnmed 110:946960

9. Syme SE, Fried JL, Strassler HE (1997) Enhanced visualization using magnification systems. J Dent Hyg 71:202-206

10. Keinan D, Nuni E, Slutzky-Goldberg I (2009) Is a C-shaped configuration possible in teeth other than mandibular molars? Quintessence Int 40:541-543

11. Kottoor J, Velmurugan N, Sudha R, Hemamalathi S (2010) Maxillary first molar with seven root canals diagnosed with cone-beam computed tomography scanning: a case report. J Endod 36:915-921

12. Tzanetakis GN, Lagoudakos TA, Kontakiotis EG (2007) Endodontic treatment of a mandibular second premolar with four canals using operating microscope. J Endod 33:318-321

13. Trotter J (1995) Das auge: Ein handbuch für augenoptiker, Neuauflageth edn. Optik Verlag Trimbach, Switzerland

14. Hettmansperger TPMJW (2011) Monographs on statistics and applied probability 119. Robust nonparametric statistical methods, 2nd edn. CRC Press, Boca Raton

15. Woo GC, Ing B (1988) Magnification devices for the presbyopic dentist. J Can Dent Assoc 54:447-449

16. Gilbert JA (1980) The dentist and the aging eye. J Mo Dent Assoc 60:22-24

17. Burton JF, Bridgman GF (1990) Presbyopia and the dentist: the effect of age on clinical vision. Int Dent J 40:303-312

18. Rawlinson A (1988) A study to investigate the visual quality of dental undergraduates using a simple screening programme. Aust Dent J 33:303-307

19. Rawlinson A (1993) A simple eyesight screening programme for dental undergraduates: results after 7 years. Aust Dent J 38:394 399 